\section{Eine Labor-}

\section{Erfolgsgeschichte "Out of Africa"}

\author{
C. Eberle
}

Die Geschichte findet in Malawi statt, und ich staune selbst immer noch über das Erreichte.

Malawi liegt im südöstlichen Teil Afrikas, mit Grenzen im Norden zu Tansania, im Westen zu Sambia und im Süden und Osten zu Mozambique. Das Land mit mehr als 10 Millionen Einwohnern weist

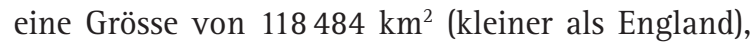
eine Länge von $840 \mathrm{~km}$ und eine Breite von $160 \mathrm{~km}$ auf. Es gehört somit zu den kleinsten, überbevölkerten Ländern Afrikas und ist leider auch ein vergessenes Land. Der grösste Anteil der Bevölkerung lebt auf dem Lande und ernährt sich von der Landwirtschaft. Die Hauptstadt, Lilongwe, mit weniger als einer halben Million Einwohnern, liegt im Zentrum, auf einer Hochebene. Die Landschaft Malawis wird vom «Great Rift Valley" und vom wunderschönen Lake Malawi beherrscht. Der See soll der drittgrösste in Afrika und der elftgrösste auf der Welt sein. Zwei bis zu 3000 m hohe Berggipfel begrenzen den Süden sowie den Norden des Landes.

Die Geschichte Malawis ist geprägt vom Leben und Werk des schottischen Arztes und Missionars David Livingston (1859-63). Erst seine Entdeckungsreise brachte der Welt Kenntnis von diesem Teil der Erde. 1907 wurde Malawi ein britisches Protektorat. Nachdem die Engländer ihre Kolonie 1964 in die Unabhängigkeit entlassen haben, litt Malawi 30 Jahre unter einer Diktatur und seit der Wegwahl des ersten Präsidenten, Dr. Banda, herrscht eine fragwürdige Demokratie. Der immer noch omnipräsente britische Einfluss führt dazu, dass neben der Landessprache Chichewa in Schulen, Wirtschaft und Regierungsgeschäften Englisch die offizielle Amtssprache ist.

Politisch, wirtschaftlich und medizinisch befindet sich Malawi in einem katastrophalen Zustand. Mehr als die Hälfte der Bevölkerung findet keine Arbeit, zum alltäglichen Geschehen gehören Kriminalität und Korruption. Der Grossteil der Bevölkerung lebt von der Landwirtschaft (Mais), Exportwaren ausser Tee und Tabak existieren nicht. In Zeiten grosser Trockenheit oder anhaltender Regenfälle kann nicht geerntet werden und Hungersnot ist die Folge davon. So geschehen dieses Jahr, die Überschwemmungen

Korrespondenz:

Charlotte Eberle

Schneckenmannstrasse 34

CH-8044 Zürich

E-mail: charlotte.eberle@freesurf.ch zerstören die Maisernte, die Infrastruktur bricht zusammen. Es fehlt an sauberem Wasser und Elektrizität, Strassen und Brücken werden weggeschwemmt. Da die Menschen mehrheitlich auf feuchtem Boden, in vom Regen ungeschützten Behausungen leben, leidet die Hygiene darunter. Das hoffnungslos überbevölkerte Land, ohne genügend Anbaufläche, ohne genügend Nahrung, kann die hungrigen Mäuler nicht mehr stopfen, und die Wälder werden kahl abgeholzt zum Zwecke der Feuerholzgewinnung.

Selbstverständlich grassieren in diesem Umfeld Krankheiten, und am stärksten trifft es die Kinder. Medizinische Hilfe ist daher dringend notwendig.

Es folgt eine Aufzeichnung der am häufigsten vorkommenden Erkrankungen:

- Malaria

- Aids

- Tuberkulose

- Lungenentzündungen

- Lebererkrankungen

- Dysenterie

- Anämie

- Meningitis

- Herzinfarkt

- Typhus

- Diabetes

- Bluthochdruck

- verschiedene Tropenerkrankungen

- natürliche und unnatürliche Unfälle

\section{Spitalwesen}

Es gibt ungefähr 18 staatliche Gesundheitseinrichtungen und ebensoviel Missionsspitäler, von denen nur die wenigstens einen genügenden medizinischen Standard nachweisen können. Es fehlt an Wissen, Technik und vor allem an den nötigen Medikamenten.

Blantyre ist die grösste Stadt, wirtschaftliches Zentrum und liegt auf 1200 m. ü. M. Das Spital ist ein Adventistenspital, das zurzeit privatwirtschaftlich geführt wird. Die treibende Kraft ist ein Schweizer Spezialist für Innere Medizin. Von ihm kommt auch der Hilferuf nach besseren Diagnosemöglichkeiten im Laborbereich. Unterstützt wird er von weiteren 5 Spezialärzten (anderen Ausländern) sowie 2-4 malawischen Assistenten, meist noch in Ausbildung. Sogar eine Zahnklinik mit einem Zahnarzt fehlt nicht. Das Spital mit 50 Betten und einem umfangreichen Ambulatorium hat einen ausgezeichneten Ruf. Die Kranken nehmen einen weiten Weg auf sich, selbst aus Mozambique reisen sie an, um die bestmögliche Hilfe zu bekommen.

Hier bin ich nun, in meinem von mir vor 4 Jahren geplanten Laborbereich. In diesem Umfeld ist es meine Aufgabe, das Labor auf Vordermann zu bringen. Die technische Infrastruktur lässt zu wünschen übrig, die elementaren Laborgeräte und Hilfsmittel fehlen. Was nun gefragt ist, sind Improvisationstalent und unbürokratische Lösungen. Zum erstenmal bin ich gefordert, mein angesammeltes Wissen von 40 Jahren Labormedizin in die Tat umzusetzen. 


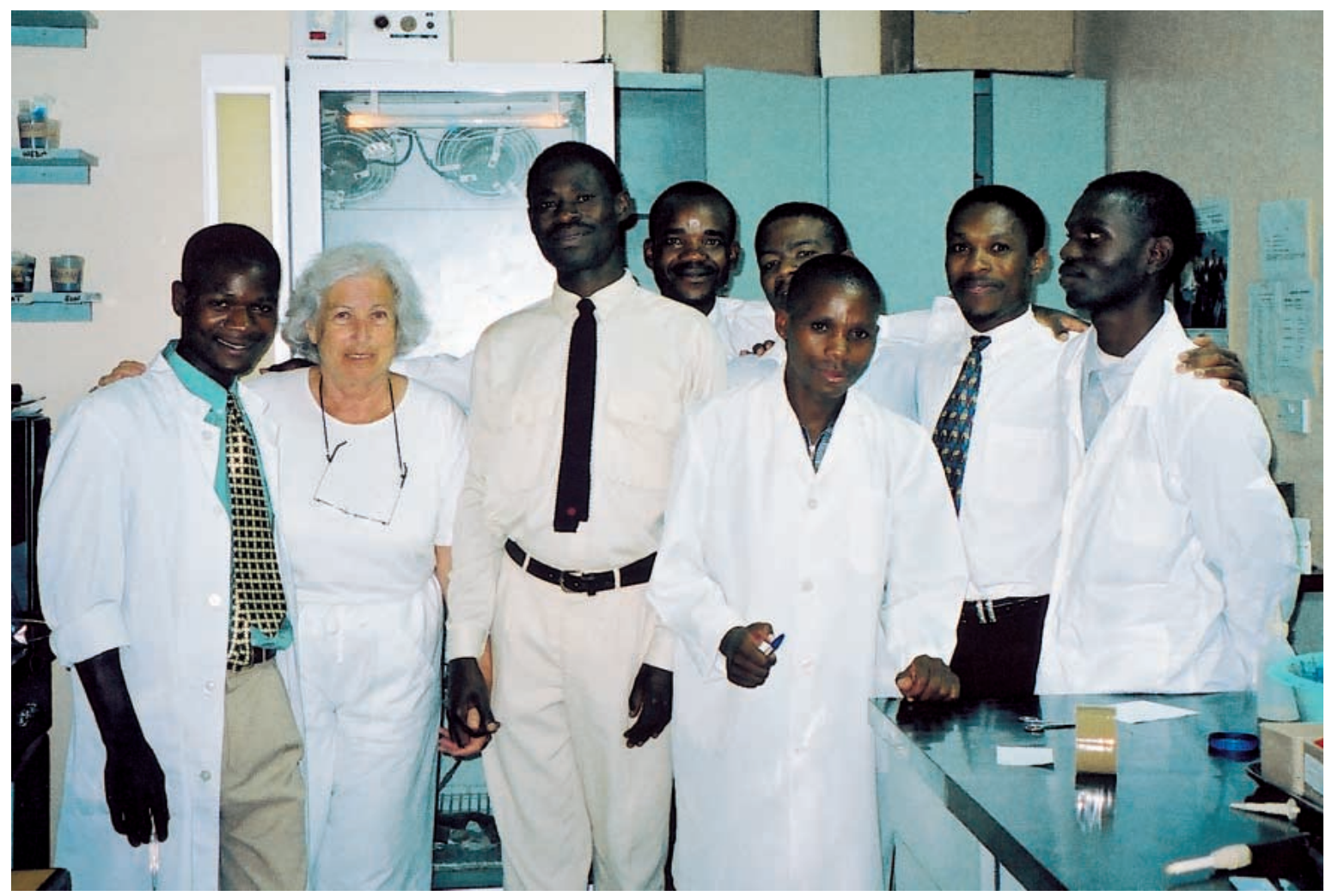

Die Hälfte meiner Besatzung!

Da ich vorbereitet war und also wusste, was auf mich zukommen würde, habe ich bereits in der Schweiz Vorarbeit geleistet. Ich habe die nötigen Kontakte mit Labordiagnostika-Unternehmen in Johannesburg (Südafrika) in die Wege geleitet und von dort bekam ich auch grosszügige Hilfe. Mein wichtigstes Anliegen war, Hilfe vor Ort zu finden, um technische Unterstützung und Einkauf von Chemikalien unbürokratisch zu ermöglichen. Die Möglichkeit, die Analysengeräte vor Ort zu reparieren, ist unabdingbar für die Genauigkeit der Resultate. Meine Erfahrungen mit gespendeten, gebrauchten Geräten, wohlmeinend, aber weit verfehlt, sind niederschmetternd. Diese Geräte arbeiten gerade mal ein paar Monate, nachher liegen sie nur funktionsuntüchtig im Wege.

Mir stehen 13-14 bestens ausgebildete Labortechniker zur Verfügung. Im Gegensatz zur Schweiz ist dies in Malawi immer noch ein Männerberuf. Gearbeitet wird beinahe rund um die Uhr, 7 Tage in der Woche. Um 22 Uhr sitzen immer noch Wartende im Ambulatorium, die Nacht verläuft sehr lebhaft.

Die Laborräumlichkeiten wurden nach meinen Skizzen und Vorstellungen eingerichtet. Ein grosser Laborraum und ein separater Raum für die Mikrobiologie. Natürlich ist bereits alles wieder zu knapp bemessen und es wird auf Vergrösserung gehofft.
Folgende Laborbereiche stehen zur Verfügung

\section{Hämatologie}

Es ist ein nicht funktionierender Coulter (sogenanntes Geschenk) vorhanden. Da wir täglich im Durchschnitt 50-60 CBC (komplettes Differentialblutbild) und beinahe ebensoviele Malariaausstriche zu bewältigen haben, ist rasche Hilfe notwendig, ansonsten die Genauigkeit der Resultate in Frage gestellt werden muss. Zwei gute Mikroskope stehen zur Verfügung und es werden mehr oder weniger gut gefärbte Blutausstriche hergestellt und in alten verkratzten Zählkammern die Blutkörperchen gezählt.

\section{Klinische Chemie}

Ein Trockenchemiegerät, von mir vor 4 Jahren in die Wege geleitet, steht bereit, sollte aber noch einer Wartung und Instruktion unterzogen werden. Aber dem stehen keine grossen Schwierigkeiten im Wege, da ja die nötigen technischen Kontakte von mir bereits hergestellt worden sind (Natrium, Kalium und $\mathrm{CO}_{2}$ werden auch damit bestimmt).

\section{Immunchemie}

FHV, Hepatitis, Bilharziosa, Typhus, (Malaria) usw. werden mit kommerziellen Kits (Schnelltests) durchgeführt. Bei zahlungskräftigen Patienten werden erweiterte immunologische Untersuchungen in Südafrika durchgeführt. Auch wir hoffen, in Zukunft Enzyme Immunoassay (EIA) durchführen zu können. 
Gerinnungsmedizin

Hier ist ein Coaguliser dringend nötig. Es wird zurzeit nach alter Sitte von Hand gehäkelt. Ich muss gestehen, es wird gar nicht schlecht durchgeführt. (Hilfe wird gerne entgegengenommen.)

\section{Transfusionsmedizin}

(sehr rudimentär); es ist noch viel Arbeit und Ausbildung nötig auf diesem Spezialgebiet. Vor allem fehlt es hier an den nötigen Reagenzien, die alle aus Südafrika importiert werden müssten, was unvorstellbar ist. In Malawi gibt es kein Nationales Blutspendezentrum.

\section{Mikrobiologie (afrikaspezifisch)}

Malawi bietet eine sehr gute Ausbildung in Mikrobiologie an, natürlich etwas veraltet wie bei uns vor 30 Jahren. Die wichtigsten Bakterienkulturen können durchgeführt werden. Da die Tuberkulose epidemisch vorhanden ist, habe ich mich aus letzterer zurückgehalten. Urin (Teststreifen), Stuhl und Sputum werden mikroskopisch, wie üblich, durchgeführt.

\section{Spezielles}

(T-Lymphozyten). Dank der finanziellen Unterstützung von Médecins Sans Frontières (MSF) können wir die Bestimmungen für CD 4 / CD 8 bei den von MSF geschickten Patienten durchführen.

\section{Allgemeines Labormaterial}

Hier fehlt es an fast allem; es hat, so lange vorrätig. Zentrifugen machen einen Riesenspektakel und vieles wird notdürftig improvisiert. Das Wegwerfmaterial für Blutentnahmen ist vorhanden, aber an den Laborpipetten und Spritzen wird gespart, was natürlich recht gefährlich ist und Kontaminationen zur Folge hat. Es wird zum Teil sehr gefährlich gearbeitet, was Menschen und die Genauigkeit betrifft. Handschuhe sind unregelmässig erhältlich. (Ich habe meine eigenen mitgebracht.) Hier sind Pipetten, Wegwerfspritzen und Handschuhe sehr sinnvolle Geschenke und sehr willkommen.

\section{Ausblick}

Nach viel Arbeit, bei der viel Humor und Geduld gefordert ist, lässt sich das Resultat meiner Bemühungen sehen. Jetzt wird speditiv auf einem ABXHämatologie-Coulter gearbeitet. Mit dem Trockenchemiegerät (Ektachem, früher Vitros) können nun 17-20 verschiedene chemische Analysen angeboten werden.

Wie sieht dies wirtschaftlich aus? Ja, da gibt es schon noch einige Fragezeichen. Die Laboruntersuchungen sind natürlich nicht gratis und aus den Einnahmen sollte sich das Labor selbst finanzieren. Trotz sehr tiefer Löhne, jedoch sehr hoher Preise der Verbrauchsmaterialien, geht es ohne Hilfe von aussen nicht.

Ohne tatkräftige Finanzspritze, vor allem aus der Schweiz, würde die Zukunft schlecht aussehen. Da wird es nach meinem Weggang Engpässe geben, denn ohne dauerndes, hartnäckiges Kämpfen und vor allem zehnmaliges Wiederholen des soeben Gesagten bewegt sich in Afrika kaum etwas. Es ist zu hoffen, dass die reichen, zahlungskräftigen Engländer, Inder und Touristen (und Entwicklungshelfer), die sich in Malawi tummeln, auch weiterhin den topmedizinischen Service des Blantyre Adventist Hospitals in Anspruch nehmen werden. Nur durch deren finanzielle Mithilfe wird die Behandlung auch für die ärmsten der Armen ermöglicht.

Der Grundstein zu einer guten Labordiagnostik ist gelegt, es besteht durchaus Aussicht auf Erfolg, denn das Personal und das Umfeld sind funktionsfähig. Die Zukunft ist weitgehend von den weiteren politischen und wirtschaftlichen Entwicklungen im Lande abhängig und damit sieht es sehr schlecht aus.

Ich wünsche meinem Laborteam alles Gute und hoffe wie im Märchen: «Wenn sie noch nicht gestorben sind, leben sie heute noch, oder wenn das Umfeld noch stimmt, arbeiten sie heute noch."

Für allfällige Spenden danken wir!

Kantonalbank Zürich, Filiale Wipkingen,

Konto-Nr. 1116-0049.091

Projekt: Blantyre-Afrika (Vreny Jaggi) 